\title{
Calculating estimates of sensitivity from group data: Pooled versus averaged estimators
}

\author{
MICHAEL J. HAUTUS \\ University of Auckland, Auckland, New Zealand
}

\begin{abstract}
Two methods of estimating the value of population $d^{\prime}$ from a group of subjects were investigated to determine the conditions under which each would be the less biased estimator. The first method, calculating $d^{\prime}$ for each subject and then averaging the estimates, yields an estimate of population $d^{\prime}$ called average $d^{\prime}$. The second method requires that data from all subjects be pooled and then an estimate of population $d^{\prime}$ calculated. This estimator is called pooled $d^{\prime}$. Monte Carlo simulations indicated that pooled $d^{\prime}$ was the less biased estimator when the number of trials per subject was fairly low, whereas average $d^{\prime}$ was the less biased estimator when the number of trials per subject was fairly high. The crossover point at which average $d^{\prime}$ becomes less biased than pooled $d^{\prime}$ is shown to depend on the value of population $d^{\prime}$, the average bias of subjects in the population, and also on the level of criterion variability within the population.
\end{abstract}

One method used to obtain an estimate of the value of population $d^{\prime}$ from a group of subjects is to first calculate a value of $d^{\prime}$ for each individual subject and then take the mean of these estimates as the population estimator. An estimate of population $d^{\prime}$ 'obtained by this method is called "average $d^{\prime}$ ". Previous advice suggests that average $d^{\prime}$ is less biased than pooled $d^{\prime}$, which is obtained using a commonly employed alternative method for estimating population $d^{\prime}$. Pooled $d^{\prime}$, as its name suggests, is obtained by pooling the data from all subjects and then estimating population $d^{\prime}$ directly from those pooled data. For an account of this topic, see Macmillan and Kaplan (1985), and Macmillan and Creelman (1991). ${ }^{1}$

At this point, consideration is given to the contexts in which an estimate of population sensitivity may be sought. An obvious context is that in which a population of subjects with common characteristics is specified, and a sample of those subjects is randomly selected so that an estimate of the average sensitivity of the entire population can be made. Less obvious is the context in which the sensitivity of only a few subjects is determined, not necessarily for the purpose of estimating the average population sensitivity. If the individual estimates of sensitivity vary considerably, it may be desirable to obtain a single statistic to summarize the average performance of that particular group of subjects. Calculating this summary statistic is formally equivalent to estimating the sensitivity of the population from which the subjects were drawn. The distinction between these two contexts has been drawn

\footnotetext{
The author would like to thank John Irwin for comments made on an earlier draft of this manuscript, and Wayne Donaldson and Doris Aaronson for suggestions made during the review process. Correspondence should be addressed to M. Hautus, Department of Psychology, The University of Auckland, Private Bag 92019, Auckland, New Zealand (e-mail: m.hautus@auckland.ac.nz).
}

to make it clear that the methods described here have broad application.

One reason for not using pooled data to estimate population $d^{\prime}$ is that the data from each individual subject reflect $t w o$ quantities: the sensitivity of the subject and the bias of the subject. The position of the datum in the ROC square indicates both bias and sensitivity. However, simply taking the average of the false alarm rates (F) and of the hit rates $(\mathrm{H})$ of several subjects does not take these two factors into account. Rather, it produces a point in ROC space that lies at the centroid of all the individual points. An estimate of sensitivity based on this centroid will tend to underestimate the true value of population $d^{\prime}$; hence, pooling is undesirable.

A second reason to avoid pooling data whenever possible is that this method provides no estimate of the variation in sensitivity between individual subjects; at least not by simple means. This makes it difficult to make statistical inferences regarding the differences between two or more pooled estimators. However, one way to overcome this difficulty is to calculate a standard error on the pooled estimate of population $d^{\prime}$ by employing a resampling scheme such as the jackknife or the bootstrap (see, e.g., Dorfman \& Berbaum, 1986, for one application of resampling schemes).

A consideration that arises for either approach to estimating population sensitivity is how to take into account the effect of extreme proportions-that is, sampled estimates of $\mathrm{F}$ or $\mathrm{H}$, which equal either zero or one. This common empirical outcome means that the inversenormal transform, which is required to calculate $d^{\prime}$, is infinite. For this reason, some form of correction must be made to the sampled data so that extreme proportions are eliminated. A useful correction is to add 0.5 to all cells in the contingency table that specifies the outcome of the experiment and, consequently, to increase the marginal totals of the table by 1 (see, e.g., Goodman, 1970; Snod- 


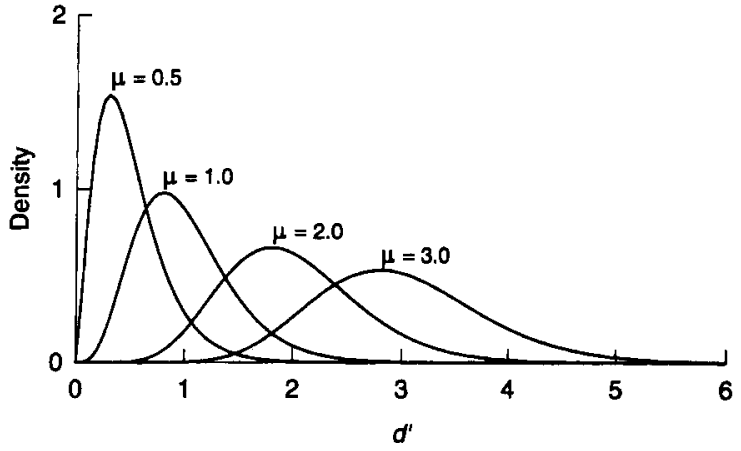

Figure 1. Gamma distributions assumed to describe the individual values of $d^{\prime}$ in the population. The four distributions represent population $d^{\prime}$ values of $0.5,1.0,2.0$, and 3.0.

grass \& Corwin, 1988). This has been termed the loglinear correction by Hautus (1995) and was shown to be less biased than the $1 /(2 N)$ correction, which adjusts extreme proportions by this factor, where $N$ is the number of trials upon which the proportion is based.

The probability of occurrence of an extreme proportion in an experiment increases as $N$ decreases, given that all other experimental parameters are held constant. Therefore, for a low enough $N$, the bias introduced into individual estimates of $d^{\prime}$ by the correction (whichever is employed) may exceed the additional bias introduced into estimates of population $d^{\prime}$ that occurs when the data of individual subjects are first pooled. If this occurs, pooled $d^{\prime}$ would be a less biased estimator than average $d^{\prime}$-at least for a small enough $N$.

\section{METHOD}

A series of Monte Carlo simulations was conducted to determine whether the bias introduced by the log-linear and $1 /(2 N)$ corrections is sufficient to make the less biased estimator of population $d^{\prime}$ move from average $d^{\prime}$ to pooled $d^{\prime}$ as $N$ decreases.

Two distributional assumptions were required to conduct the simulations. First, an assumption was made about the distribution of the sensitivity of individuals in a population. Although the mathematical theory behind signal detection theory allows $d^{\prime}$ to take on any real value, a negative value of $d^{\prime}$ can be treated as a mislabeling of the stimulus events by the subject. (This does not rule out the possibility of obtaining an estimate of $d^{\prime}$ that is negative. However, such an estimate is due to sampling variability.) Given that $d^{\prime}$ can range from zero to infinity, a gamma distribution was selected. The mean of the distribution was set equal to population $d^{\prime}$ and took on four values for this study: $M\left(d^{\prime}\right)=0.5,1.0,2.0$, and 3.0. The standard deviation of the distribution was set to one fifth of the value of population $d^{\prime}$. The four distributions are illustrated in Figure 1. It is obvious that these distributions are skewed considerably. Consideration is given later to the effects of this asymmetry.

Second, an assumption was made about the distribution of the bias of individuals in a population. The measure of bias employed was the familiar $c$, which is defined by the equation $c=-1 / 2(z(H)$ $+z(F))$, where $z(\cdot)$ is the inverse-normal transform. This measure corresponds to the distance between the criterion and the point midway between the means of the noise and signal distributions. The midpoint is at $d^{\prime} / 2$ if the mean of the noise distribution is zero. ${ }^{2}$ For these simulations, $c$ was assumed to be normally distributed with the standard deviation adjusted between $S D(c)=0.2$ and $S D(c)=$ 1.0 , in steps of 0.2 . A Gaussian assumption was made because there is no evidence, or any reason, to suggest that the distribution of biases in a population is skewed, especially for low levels of bias.

Two more parameters were manipulated: the number of trials, $N$, upon which each individual estimate was based and the number of subjects included in the study. $N$ varied in integral powers of two between 2 and 512 . The number of subjects was $1,2,3,4,5,10,20$, or 40 . It is worth noting that there are a number of areas of research in which small numbers of trials (5-20 trials per subject) are employed, such as language and memory, hemisphericity, and mental rotation, to name just a few. These areas can be contrasted with standard psychophysics, in which more than 100 trials per subject are routinely collected. To compensate, areas using few trials per subject often employ more subjects than those using many trials per subject. Thus, some of the parameter combinations investigated in this study (e.g., small numbers of both subjects and trials) would be used only rarely in practice. They are included so that a better picture of the relationships among all of the parameters can be obtained.

Each simulated experiment was defined by a combination of the four parameters manipulated. Each experiment was repeated 10,000 times and the mean of the obtained estimates of population $d^{\prime}$ was determined each time. For each experiment, estimates of population $d^{\prime}$ were calculated by both of the methods described above. All possible combinations of the four parameters were investigated twice: once with the $1 /(2 N)$ correction and again with the log-linear correction.
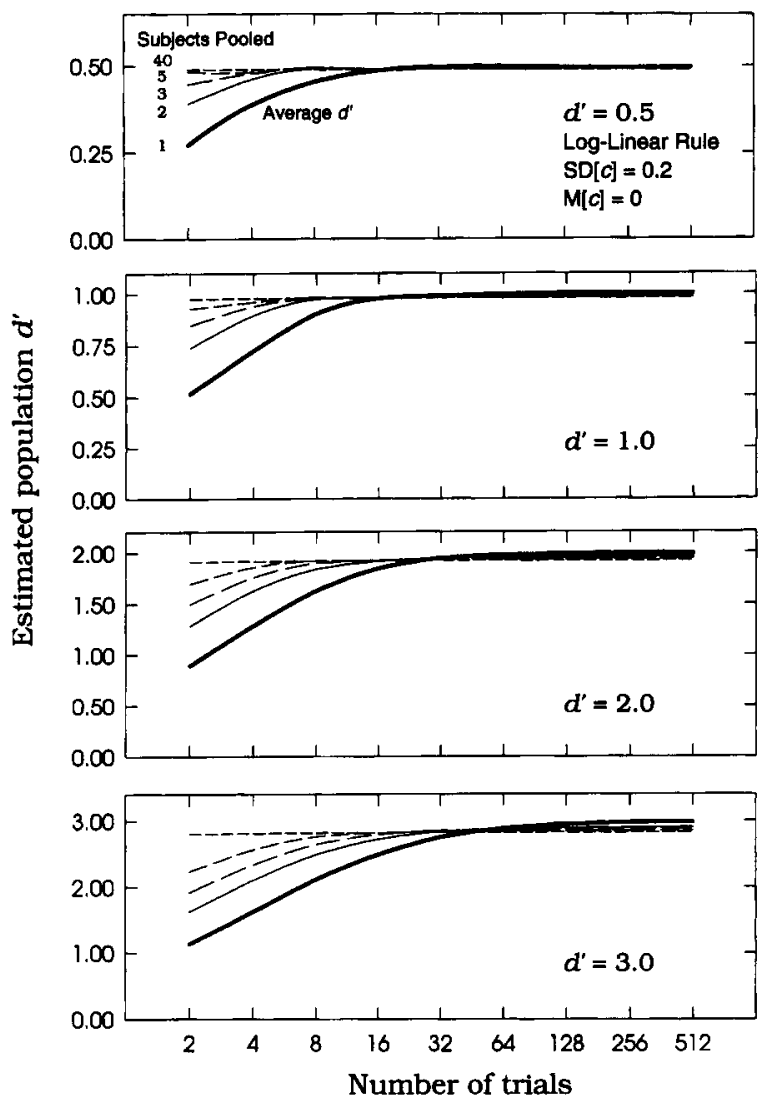

Figure 2. Average $d^{\prime}$ (thick solid line) and pooled $d^{\prime}$ (thin lines) as a function of $N$, population $d^{\prime}$, and number of subjects per group (shown to the left of each line) when the log-linear correction was applied. The criterion, $c$, is assumed to have $M(c)=0$ and $S D(c)=0.2$ 

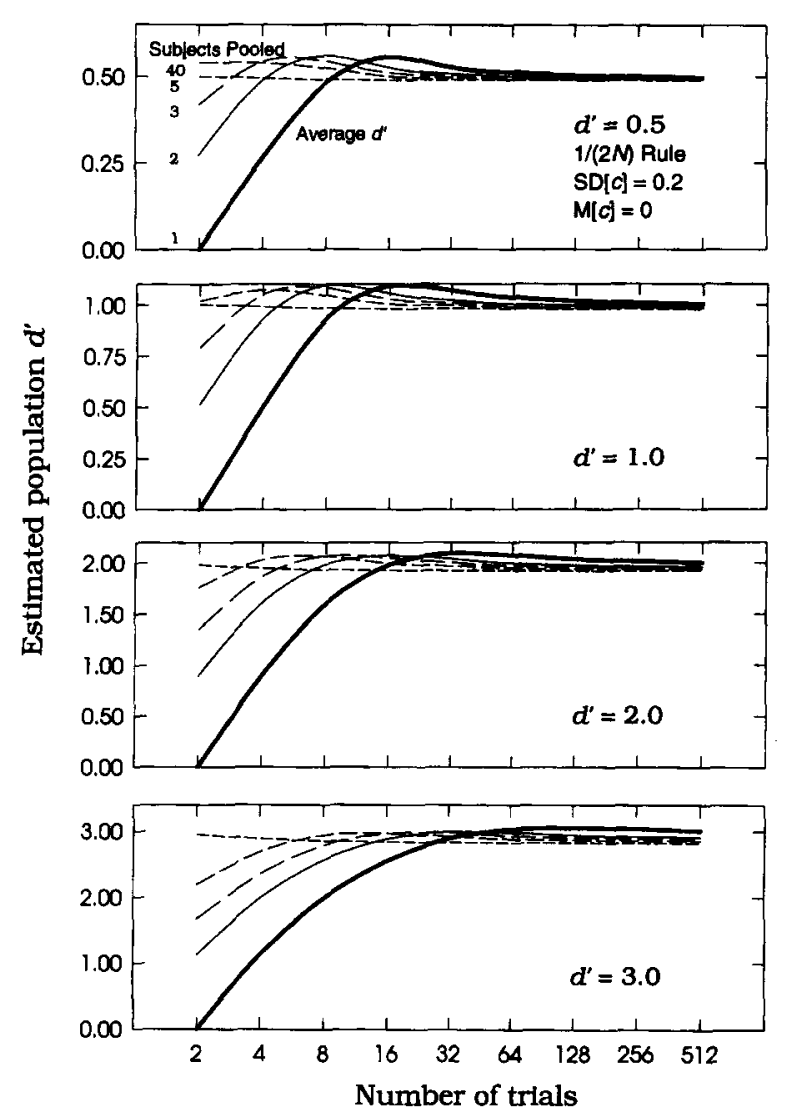

Figure 3. Average $d^{\prime}$ (thick solid line) and pooled $d^{\prime}$ (thin lines) as a function of $N$, population $d^{\prime}$, and number of subjects per group (shown to the left of each line) when the $1 /(2 N)$ correction was applied. The criterion, $c$, is assumed to have $M(c)=0$ and $S D(c)=0.2$.

\section{RESULTS}

Figures 2 through 7 illustrate estimates of population $d^{\prime}$ for a wide range of parameter values. The four panels in each figure show the results obtained for the different values of population $d^{\prime}$-that is, $d^{\prime}=0.5,1.0,2.0$, and 3.0. The thick curve in each panel runs through the estimates of population $d^{\prime}$ obtained by averaging individual estimates of $d^{\prime}$ - that is, average $d^{\prime}$. These curves were drawn thicker to indicate that they are the curves obtained irrespective of how many subjects were in the group. Trivially, these curves were also obtained for pooled $d^{\prime}$ when there was only 1 subject in the group. The broken curves in each panel pass through the estimates of population $d^{\prime}$ obtained by first pooling the data-that is, pooled $d^{\prime}$. The number toward the left-hand end of each curve in the top panel of each figure indicates the number of subjects in the group represented by that curve.

Figure 2 illustrates estimates of population $d^{\prime}$ for a range of parameters when $c$ is normally distributed with $S D(c)=0.2$. Figure 2 is based on the log-linear correction. Figure 3 has the same parameters as Figure 2; how- ever, the $1 /(2 N)$ correction was employed. Apparent in each panel of Figure 2 is a value of $N$ below which pooled $d$ ' produced a less biased estimate of the population parameter than average $d^{\prime}$. The crossover point, in terms of $N$, increases gradually as a function of $d^{\prime}$ : from about $N=17$, when $d^{\prime}=0.5$, to about $N=57$, when $d^{\prime}=3$. The $1 /(2 N)$ correction has a tendency to produce estimates of population $d^{\prime}$ that first underestimate and then overestimate the true value before asymptotically converging to population $d^{\prime}$ as $N$ increases (see Hautus, 1995). This is reflected in Figure 3. The implication of this finding is that the crossover point may not be informative regarding which method of estimating population $d^{\prime}$ is less biased for a particular value of $N$. For example, in the top panel of Figure 3 , there is a region to the right of the crossover point within which pooled $d^{\prime}$ is less biased than average $d^{\prime}$, especially for smaller group sizes.

The results depicted in Figures 2 and 3 are interesting, yet some may question whether criterion variability in this case is as large as that encountered in practice. Figures 4 and 5 illustrate data for the same parameters except that $c$ is normally distributed with $S D(c)=1.0$, a

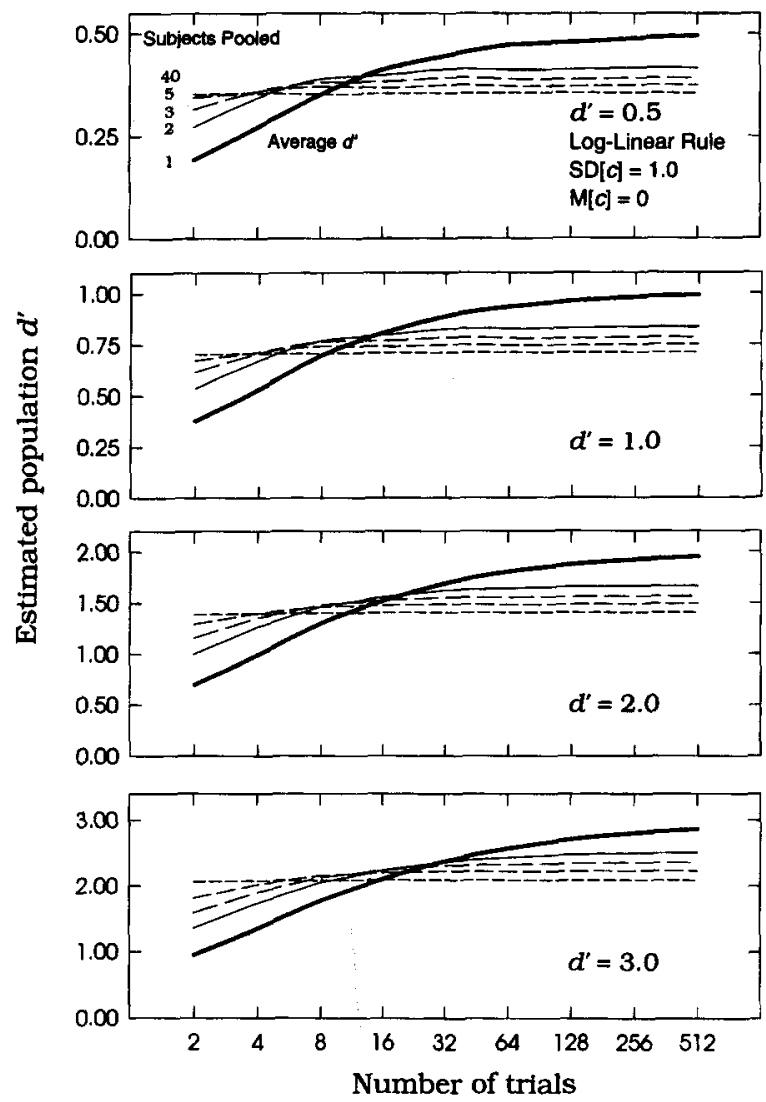

Figure 4. Average $d^{\prime}$ (thick solid line) and pooled $d^{\prime}$ (thin lines) as a function of $N$, population $d^{\prime}$, and number of subjects per group (shown to the left of each line) when the log-linear correction was applied. The criterion, $c$, is assumed to have $M(c)=0$ and $S D(c)=1.0$. 

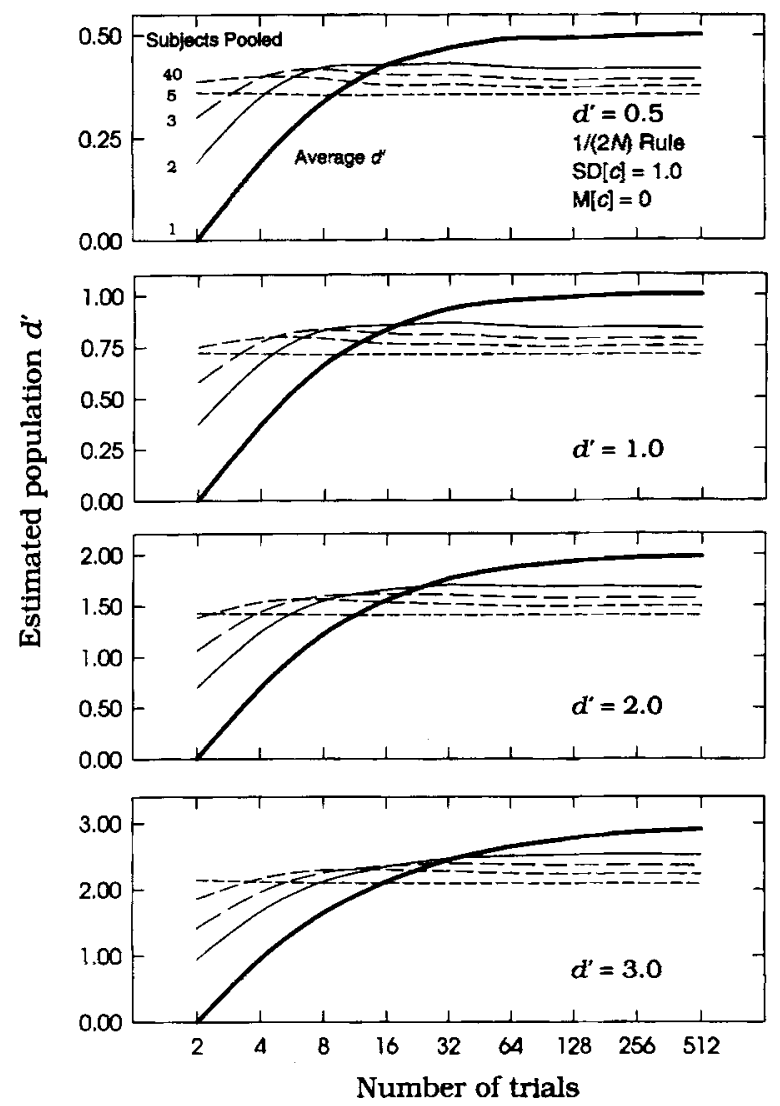

Figure 5. Average $d^{\prime}$ (thick solid line) and pooled $d^{\prime}$ (thin lines) as a function of $N$, population $d^{\prime}$, and number of subjects per group (shown to the left of each line) when the $1 /(2 N)$ correction was applied. The criterion, $c$, is assumed to have $M(c)=0$ and $S D(c)=1.0$.

rather extreme value. However, the general pattern is the same as that for Figures 2 and $3 .{ }^{3}$ The crossover points occur at about 10 trials, when $d^{\prime}=0.5$, and at about 25 trials when $d^{\prime}=3$.

So far the simulations have included only a population of subjects who were, on the average, unbiased. Figures 6 and 7 illustrate estimates of population $d^{\prime}$ for the same parameters as those for Figures 2 and 3 except that the criterion was displaced by 1 standard deviation-that is, $c=1$. The two figures are similar except that the more biased criteria caused the crossovers to occur at higher values of $N$. This follows from the finding (Hautus, 1995) that the bias introduced by either correction is least for unbiased observers (at least for a signal detection theory model that implies a symmetrical ROC curve). Thus, for more biased observers, the biasing effect of the correction is larger on each individual estimate of $d^{\prime}$, and hence average $d^{\prime}$ is also more biased.

Tables 1 and 2 give the approximate trial number, $N$, at which the crossover occurred for a variety of different population biases and group sizes when $S D(c)=0.2$, for each of the two corrections. The group sizes in these tables are different from those employed in the earlier sim- ulations. This change of parameter values allows a better description of how the crossovers varied as a function of group size. Therefore, the only overlap between Tables 1 and 2 and Figures 2 and 3 occurred for Group Size 2. It is important to remember that the crossovers reported in Table 2 may not be good indicators of which method of estimation is less biased. This is true even though the crossovers tended to occur at smaller values of $N$ than did their equivalents in Table 1.

Consideration is now given to the effect of the distributional assumptions on these simulations. The asymmetry of the distributions in Figure 1 (and more generally, the assumption of gamma distributions) suggests that some of the observed biases may be a consequence of the choice of distribution. Furthermore, the assumed normality of the distribution of $c$ may also have influenced the outcome. For these reasons, the sensitivity of the outcomes of the simulations to breaches in the distributional assumptions was assessed. The simulations were repeated under the assumption that the sensitivity of individuals in the population is normally distributed (thereby eliminating the effect of the asymmetry apparent in Figure 1) and that the distribution of $c$ in the population is negative ex-

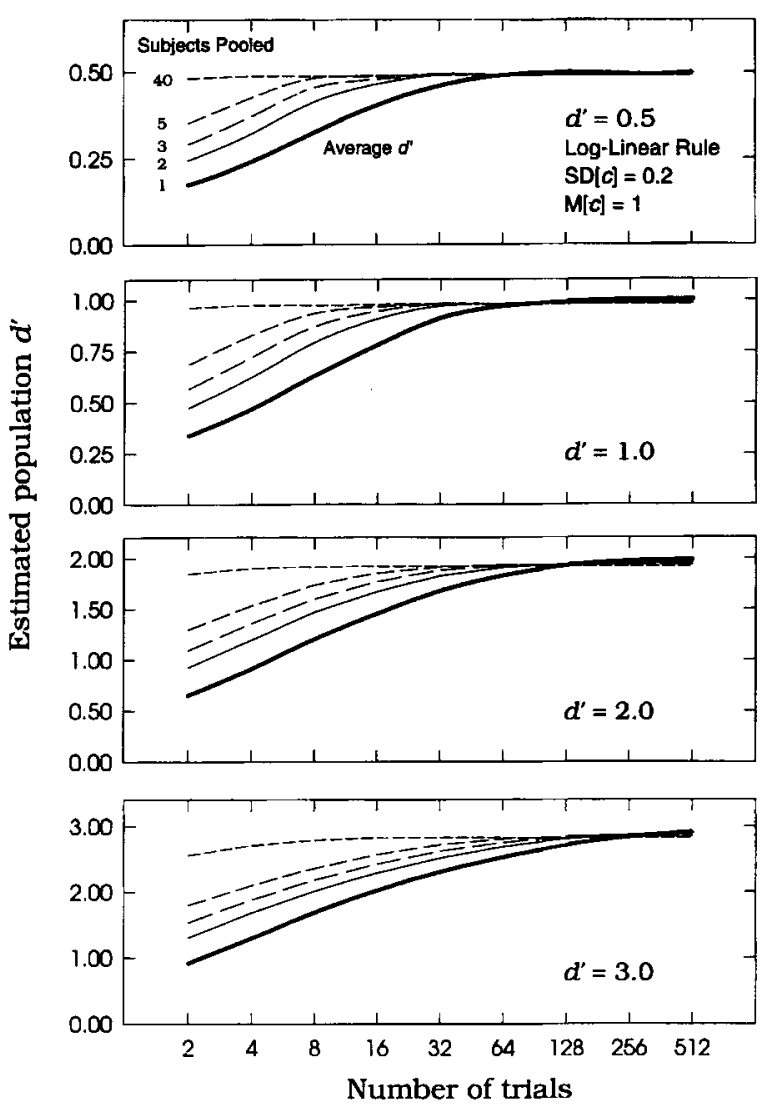

Figure 6. Average $d^{\prime}$ (thick solid line) and pooled $d^{\prime}$ (thin lines) as a function of $N$, population $d^{\prime}$, and number of subjects per group (shown to the left of each line) when the log-linear correction was applied. The criterion, $c$, is assumed to have $M(c)=1.0$ and $S D(c)=0.2$. 

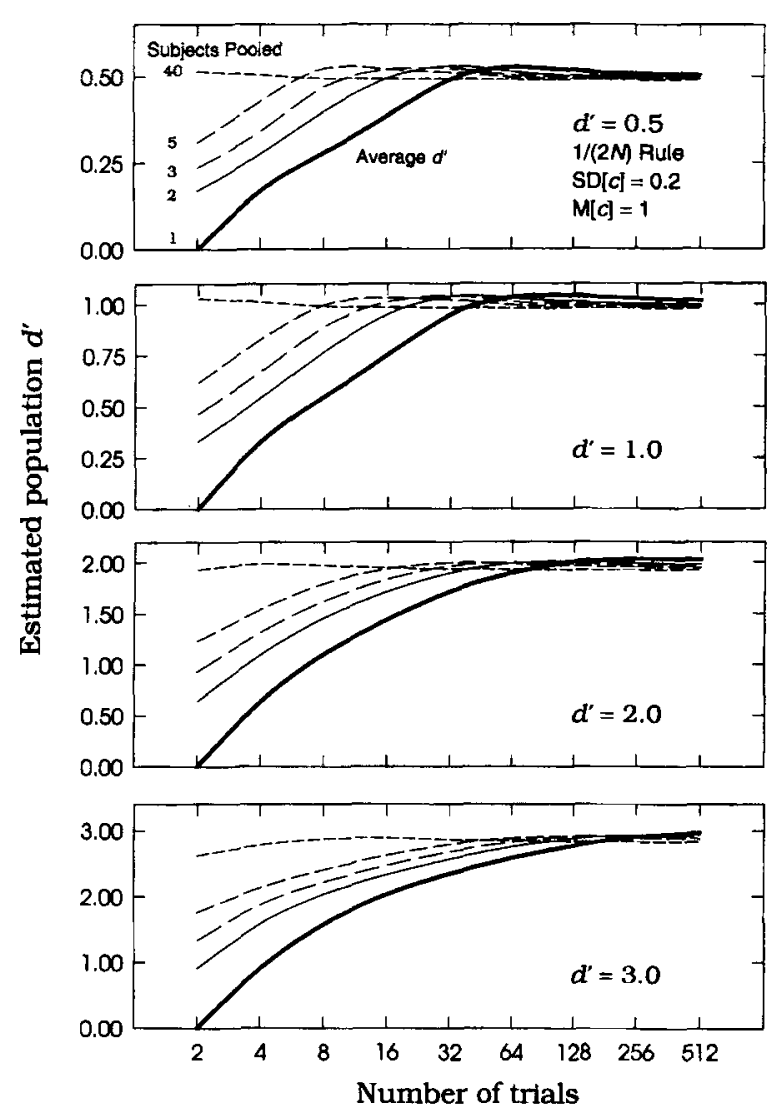

Figure 7. Average $d^{\prime}$ (thick solid line) and pooled $d^{\prime}$ (thin lines) as a function of $N$, population $d^{\prime}$, and number of subjects per group (shown to the left of each line) when the $1 /(2 N)$ correction was applied. The criterion, $c$, is assumed to have $M(c)=1.0$ and $S D(c)=0.2$.

ponential (thereby introducing considerable asymmetry into this distribution). The distributions were given the same means and variances as their earlier counterparts. The results based on normal $\left(d^{\prime}\right)$ and negative exponential $(c)$ distributions were almost identical to those obtained using the more theoretically desirable gamma $\left(d^{\prime}\right)$ and normal $(c)$ distributions. The results obtained using the $\log$-linear correction are illustrated in Figure 8 for the case where $S D(c)=0.2$, and in Figure 9 for $S D(c)=1.0$.

Figure 8 can be compared directly with Figure 2, and Figure 9 with Figure 4. Each of the figures is based on 180 points. The maximum deviation between Figures 2 and 8 of $-4.6 \%$ occurs at $d^{\prime}=0.5$ with 1 subject and two trials. The average deviation over all points between these two figures is $0.2 \%$. The differences between Figures 4 and 9 are greater. The maximum deviation is $8.7 \%$, with deviations of a similar magnitude occurring at many different parameter combinations. The average deviation of $5.6 \%$ indicates that the curves in Figure 9 lie closer to population $d^{\prime}$ than do those in Figure 4. The similarity between the figures, which are based on different assumptions, especially for the less extreme case of $S D(c)=0.2$, indi- cates that the results reported earlier are relatively insensitive to the assumptions imposed.

\section{DISCUSSION}

For researchers engaged in traditional psychophysical investigations, in which large numbers of trials (i.e., $N$ greater than about 100) are collected from a few subjects, the less biased method for calculating population $d^{\prime}$ is to calculate $d^{\prime}$ separately for each subject and then to take the average of the calculated $d^{\prime}$. This is the approach usually preferred in the literature (e.g., Macmillan \& Kaplan, 1985). The present results indicate that for large numbers of trials, it does not make much difference to the outcome whether the $1 /(2 N)$ or the log-linear correction is employed. One situation in which the efficacy of this approach needs careful checking, even with large numbers of trials, is that in which the subjects exhibit considerable bias. Pooled $d^{\prime}$ may, in some of these cases, be less biased than average $d^{\prime}$. However, this outcome would be the exception rather than the rule in most practical applications, in which more than about 100 trials are collected from each subject.

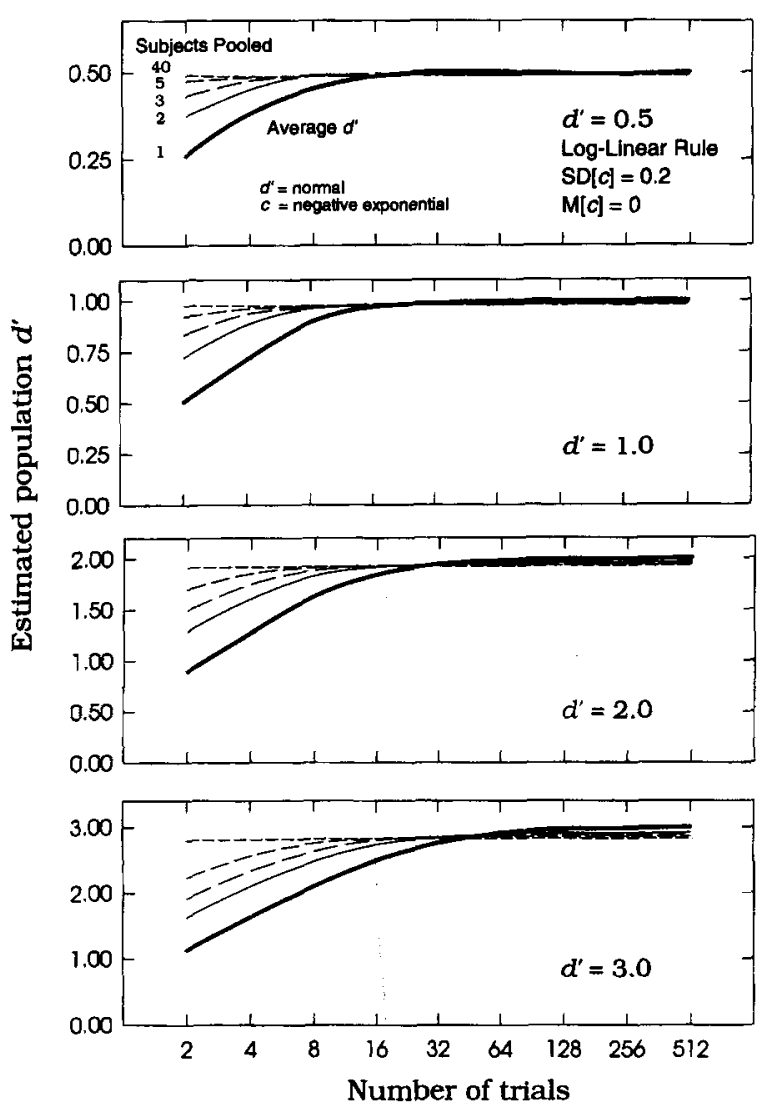

Figure 8. The parameters used in these simulations were the same as those used to obtain Figure 2 . However, the assumptions were breached fairly severely; that is, $d^{\prime}$ was assumed to be normally distributed, and $c$ was assumed to be distributed as a negative exponential. 


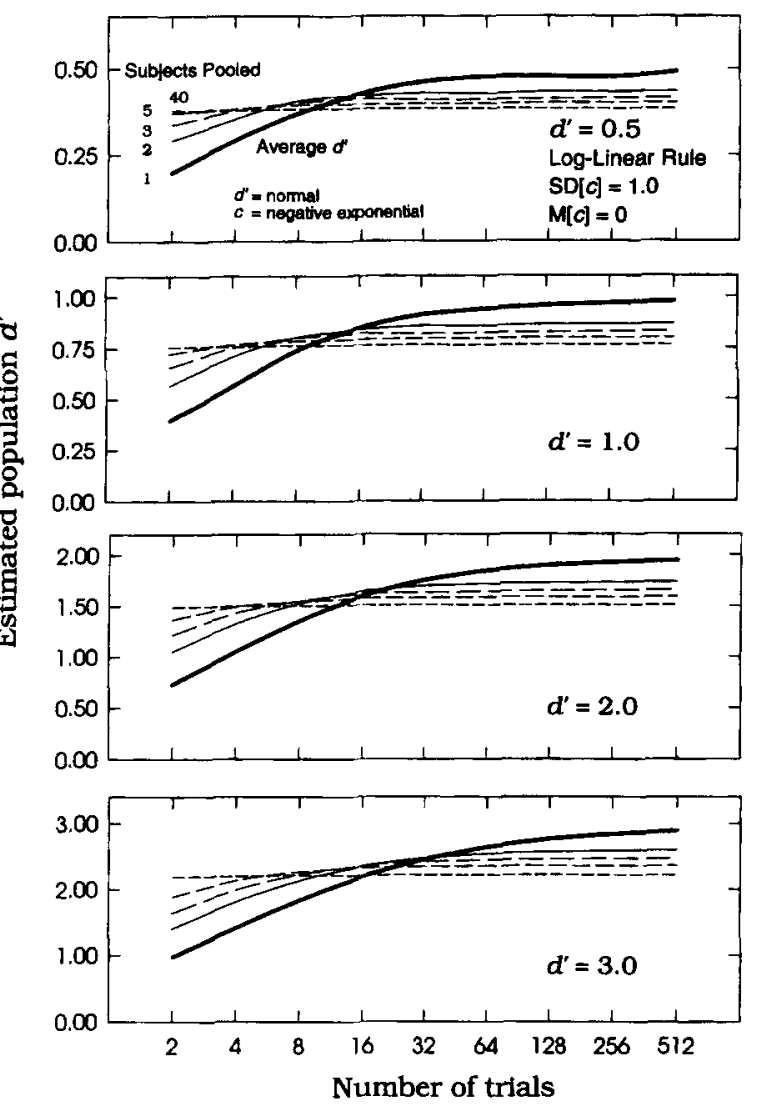

Figure 9. The parameters used in these simulations were the same as those used to obtain Figure 4. As in Figure 8, the assumptions made in earlier calculations were breached; that is, $d^{\prime}$ was assumed to be normally distributed, and $c$ was assumed to be distributed as a negative exponential.

However, for research in which only a few trials are collected from each subject, the less biased method is to first pool the data from all subjects and then to calculate $d^{\prime}$ from the pooled data. Researchers in fields such as memory, cognition, neuropsychology, or the sensory evaluation of foods and beverages would typically employ small numbers of trials. For these situations, the results indicate clearly that the log-linear correction provides the less biased estimators.

All of the results obtained in these simulations lend themselves to a very simple rule of thumb that has utility in all situations as long as the log-linear correction has been applied. The rule is as follows: Calculate both average $d^{\prime}$ and pooled $d^{\prime}$. Whichever has the highest value also has the greatest likelihood of being the less biased estimator of population $d^{\prime}$. This rule of thumb holds because estimates of population $d^{\prime}$ calculated using the loglinear correction asymptotically approach the true value of population $d^{\prime}$ from below. Thus, the estimator that provides the largest value will have the greatest likelihood of being the less biased.
This rule does not hold if the $1 /(2 N)$ correction has been applied. This is because, as can be seen in Figures 3,5 , and 7 , the $1 /(2 N)$ correction can overestimate the true value of population $d^{\prime}$. This problem is clearest in the top two panels of Figures 3 and 7, where the largest valued estimator is average $d^{\prime}$, which is also the more biased estimator.

Table 1

Approximate Trial Number Below Which Pooled $d^{\prime}$ Is a Less Biased Estimator of Population $d^{\prime}$ Than Averaged $d^{\prime}$

\begin{tabular}{|c|c|c|c|c|c|c|}
\hline \multirow[b]{2}{*}{$n$} & \multirow[b]{2}{*}{ Population $d^{\prime}$} & \multicolumn{5}{|c|}{ Mean Population Criterion (c) } \\
\hline & & 0.0 & 0.5 & 1.0 & 1.5 & 2.0 \\
\hline \multirow[t]{4}{*}{2} & 0.5 & 17 & 34 & 62 & 185 & 700 \\
\hline & 1.0 & 22 & 36 & 90 & 278 & $>1,000$ \\
\hline & 2.0 & 32 & 57 & 166 & 630 & $>1,000$ \\
\hline & 3.0 & 57 & 97 & 345 & $>1,000$ & $>1,000$ \\
\hline \multirow[t]{4}{*}{4} & 0.5 & 20 & 30 & 66 & 161 & 568 \\
\hline & 1.0 & 22 & 34 & 82 & 230 & 864 \\
\hline & 2.0 & 28 & 50 & 142 & 513 & $>1,000$ \\
\hline & 3.0 & 48 & 86 & 296 & $>1,000$ & $>1,000$ \\
\hline \multirow[t]{4}{*}{8} & 0.5 & 20 & 30 & 62 & 161 & 558 \\
\hline & 1.0 & 22 & 32 & 73 & 218 & 833 \\
\hline & 2.0 & 28 & 46 & 128 & 468 & $>1,000$ \\
\hline & 3.0 & 46 & 78 & 257 & $>1,000$ & $>1,000$ \\
\hline \multirow[t]{4}{*}{16} & 0.5 & 20 & 28 & 62 & 153 & 554 \\
\hline & 1.0 & 22 & 32 & 73 & 214 & 768 \\
\hline & 2.0 & 28 & 46 & 124 & 446 & $>1,000$ \\
\hline & 3.0 & 44 & 74 & 250 & $>1,000$ & $>1,000$ \\
\hline \multirow[t]{4}{*}{32} & 0.5 & 20 & 30 & 62 & 161 & 553 \\
\hline & 1.0 & 22 & 32 & 73 & 212 & 768 \\
\hline & 2.0 & 26 & 44 & 122 & 436 & $>1,000$ \\
\hline & 3.0 & 44 & 72 & 236 & $>1,000$ & $>1,000$ \\
\hline
\end{tabular}

Note - Crossovers are given for several different combinations of population characteristics. The log-linear correction was applied.

Table 2

Approximate Trial Number Below Which Pooled $d^{\prime}$ Is Likely to Be a Less Biased Estimator of population $d^{\prime}$ Than Averaged $d^{\prime}$ (See Text for an Explanation of This Qualification)

\begin{tabular}{rrrrrrr}
\hline & & \multicolumn{5}{c}{ Mean Population Criterion $(c)$} \\
\cline { 3 - 7 }$n$ & Population $d^{\prime}$ & 0.0 & 0.5 & 1.0 & 1.5 & \multicolumn{1}{c}{2.0} \\
\hline 2 & 0.5 & 12 & 20 & 48 & 129 & 450 \\
& 1.0 & 14 & 23 & 57 & 180 & 682 \\
& 2.0 & 24 & 40 & 138 & 513 & $>1,000$ \\
& 3.0 & 48 & 81 & 329 & $>1,000$ & $>1,000$ \\
4 & 0.5 & 10 & 16 & 38 & 103 & 329 \\
& 1.0 & 12 & 19 & 47 & 139 & 512 \\
& 2.0 & 18 & 31 & 102 & 369 & $>1,000$ \\
& 3.0 & 36 & 65 & 257 & $>1,000$ & $>1,000$ \\
8 & 0.5 & 10 & 15 & 35 & 92 & 306 \\
& 1.0 & 10 & 16 & 42 & 122 & 439 \\
& 2.0 & 16 & 26 & 85 & 316 & $>1,000$ \\
& 3.0 & 31 & 53 & 209 & 961 & $>1,000$ \\
16 & 0.5 & 10 & 14 & 33 & 88 & 280 \\
& 1.0 & 10 & 16 & 40 & 114 & 410 \\
& 2.0 & 15 & 24 & 77 & 284 & $>1,000$ \\
& 3.0 & 28 & 47 & 185 & 832 & $>1,000$ \\
32 & 0.5 & 9 & 14 & 32 & 86 & 275 \\
& 1.0 & 10 & 15 & 38 & 110 & 393 \\
& 2.0 & 15 & 23 & 74 & 274 & $>1.000$ \\
& 3.0 & 27 & 44 & 172 & 781 & $>1,000$ \\
& 1.0 & &
\end{tabular}

Note-Crossovers are given for several different combinations of population characteristics. The $1 /(2 N)$ correction was applied. 


\section{REFERENCES}

Dorfman, D. D., \& BeRbaUM, K. S. (1986). RSCORE-J: Pooled ratingmethod data: A computer program for analyzing pooled ROC curves. Behavior Research Methods, Instruments, \& Computers, 18, 452-462.

GoOdMAN, L. A. (1970). The multivariate analysis of qualitative data: Interactions among multiple classifications. Journal of the American Statistical Association, 65, 226-256.

Hautus, M. J. (1995). Corrections for extreme proportions and their biasing effects on estimated values of $d^{\prime}$. Behavior Research Methods, Instruments, \& Computers, 26, 46-51.

Macmillan, N. A., \& Creelman, C. D. (1991). Detection theory: A user's guide. Cambridge: Cambridge University Press.

Macmillan, N. A., \& KaPlan, H. L. (1985). Detection theory analysis of group data: Estimating sensitivity from average hit and falsealarm rates. Psychological Bulletin, 98, 185-199.

Snodgrass, J. G., \& Corwin, J. (1988). Pragmatics of measuring recognition memory: Applications to dementia and amnesia. Journal of Experimental Psychology: General, 117, 34-50.

\section{NOTES}

1. The index $d^{\prime}$ implies a symmetrical ROC in the unit probability square. This index also implies an ROC that is a straight line with unit slope when plotted on inverse-normal coordinates. The methods outlined in this manuscript apply only to this equal-variance normal model. Alternative methods, such as those outlined by Dorfman and Berbaum (1986), need to be used for asymmetrical ROCs such as those based on an unequal-variance normal model.

2. $\log (\beta)$ is another measure of bias that is often reported in the literature. A simple relationship exists between the two measures, $\log (\beta)=c d^{\prime}$ (Macmillan \& Creelman, 1991, p. 40). Therefore the results reported in this paper apply equally for both measures.

3. The difference between the figures obtained for different levels of criterion variability is the degree to which population $d^{\prime}$ is underestimated at any particular $N$. This is readily apparent from the top two panels in Figures 4 and 5 when compared with those in Figures 2 and 3. Overall, larger criterion variability produces larger biases in estimates of population $d^{\prime}$.

(Manuscript received February 19, 1996;

revision accepted for publication September 23, 1996.) 